\title{
Seroprevalencia de Legionella pneumophila en Santiago: evidencias de exposición durante la infancia
}

\author{
Teresa Lobos M.' ; Pablo A. Vial C. ${ }^{\text {; }}$ Paula Piemonte L. ${ }^{3}$; \\ Jimena Ovalle P.4; Rodrigo Morcno B. ${ }^{\text {; }}$; Callerina Ferreccio R. ${ }^{6}$ \\ Evidence of Legionella pneumophila infection \\ in chilean subjects under twenty years of age
}

\begin{abstract}
Evidence of pasl exposure to t. preuncptita was evaluated in a 120 chilcan subjects less than 20 years old shatified by socicaconomic bockground and age. Serum samples were analyzed for l. preumophila ontlbadies by indired immunofluorescence using serogrcup 1 to 0 ontigens: lilers equal or greoter than 1:04 were considered positive. Overall, 12 out of 120 samples ( $10 \%$; were positive for $t$. pneumophilc antibodies and different prevalence roles were recorded anong individuals of low $10 / 40)$, middle $(2,40 ; 5 \%)$ and high $110 / 40 ; 25 \%)$ socioeconomic groups. The highest liters observed in this healthy population based survey were $1: 128$. High socioeconomic bockground is associated with highest prevalence of anti-1. pneumophita antibedies in chilean population.
\end{abstract}

[Key words: legionello pneumophita, fluorescence immunoassay, antibodies, prevolence.\}

La enfermedad del legionario, clínicamente caracterizada como una neumonia alipica, fuc descrita por primera vez en 1976', cl año siguiente se descubrió su agente etiológico ${ }^{2}, L e-$ gionella pneumophila, un bacilo Gram negativo, aerobio obligado, de crecimiento fastidioso. Desde entonces se han descrito al menos $20 \mathrm{es}$. pecies del género Legionella y 12 serogrupos de L. pneumophila. Las bacterias de este género lienen una amplia distribución y alcanzan altas concentraciones en ambientes acuáticos tales como agua de lagos y ríos, agua polable, termos de agua caliente y sistcmas de crfriamiento de aire acondicionado ${ }^{3}$.

1. Departamento de Medicina y Centro de Investigaciones Médicas, Universidad Católica de Chile.

2. Departamento de Pediatría y Centro de Investigaciones Médicas, Universidad Calólica de Chile.

3. Becada en Microbiologia, Universidad de Chile.

4. Centro de Investigaciones Médicas, Universidad Católica de Chile.

5. Depanamento de Enfermedades Respiralorias, Universidad Católica de Chile.

6. GREDIS (Grupo para el Desarrollo de Investigución en Salud).

Este proyecto contó con financiamiento del proyecto IDUC-91 de Child Health Foundation.
La epidemiología de L. pnetumophila y su importancia como palógeno humano ha sido estudiada principalmente por evaluaciones serológicas ${ }^{3-6}$, determinándose que 2 a $30 \%$ de la población adulta ha tcnido exposición a estc agente evidenciada por presencia de anticuerpos específicos y que clla es infrecuente en la población pediátrica. En coincidencia con esta información se ha determinado que $L$. pneumophila es el agente causal de 5 a $10 \%$ de las neumonias del adulto y sólo $1 \%$ de las que ocurren en menores de 15 años $\mathrm{s}^{5-9}$.

Considerando el creciente interés por conocer la magnitud epidemiológica de los patógenos que producen neumonia y la creciente demanda por lécnicas de diagnostico ctiológico de estas infecciones, el presente estudio tuvo por objetivo determinar, mediante un marcador serológico, la exposición a L. pneumophila en la población mcnor de 20 años de Santiago de Chile y cstandarizar un ensayo scrológico que permita identificar los casos de infección aguda.

\section{Población y métodos}

Para la detcrminación de secoprevalencia se utilizó una muestra aleatoria de una colección de sueros disponible en 
cl Centro de Investigaciones Médicas. Estos sueros fueron oblenidos durante el año 1991 en un mucstreo de la pobla. ción de Santiago realizado por visitas al hogar, en la que se inciuyó una encuesta socioeconónica. de hábílos higénicos y de salud. El único crilerio de exclusión fue haber padecido una infeccion respiratoria baja en los tres meses previos a la toma de la muestra de sangre. La delerminación de nivel socioeconómico (NSE) se realizó mediante escala de Grafar modificada. Se incluyeron 120 individuos, tistribuyéndose 40 en cada NSE (bajo, medio y alto). En cada subgrupo de NSE, la muestra se distribuyó proporcionalmente en cuatro grupos de edades: 0 a 5,6 a 10,11 a 15 y 16 a 20 años.

$L a$ detección de anticuerpos anti- $L$. pneumophila se realizs con la técnica de inmunofluorescencia indirecta, utilizando antígenos polivalentes de scrogrupos 1 a 6 (Organon Teknika, Holanda). Las muestras fueron iniciatmente analizadas en dilución 1:8 y aquellas que dieron resu]ıado posilivo fueron tituladas. Puesto que cl ensayo liene reacciones inespecíficas a títulos bajos, se eligí el de 1:64 como valor de corte para desiguar una mucsisa como positiva ${ }^{10}$

\section{Resultados}

En el conjunto de las 120 muestras analizadas, doce dieron resultados positivos para anticuerpos anti-L. pneumophila, dos de las cualcs corresponden a personas de NSE medio y diez al nivel alto (tabla 1). La prevalencia en NSE alto es significativamente mayor que la de los NSE medio y bajo $(\mathrm{p}=0,02$ y $\mathrm{p}=0,01$, respectivamente). La seroprevalencia por grupo de cdades muestra que la exposición al agente comienza a aparecer después de los 5 años de vida (tabla 2), sin encontrarse diferencias significativas en los grupos etarios mayores.

La distribución de los títulos obtenidos en las 120 muestras analizadas fue $1: 8$ o menores en 61 $(50,8 \%)$ de ellas, $1: 16$ en $44(36,7 \%), 1: 32$ en 3 $(2,5 \%)$ y, como hemos señalado, 1:64 o mayor en 12 (10\%) de los casos, siendo cl título máximo observado $1: 128$, en 5 de estas últimas.

\section{Comentario}

Este estudio muestra una seroprevalencia global de $L$. pneumophila de $10 \%$ en la población menor de 20 años. Este resultado no es significalivamente diferente, como podría haberse esperado, de la seroprevalencia de $5 \%$ encontrada en 100 adultos en Santiago ${ }^{11}$ con la misma técnica serológica, idéntico valor de corte y distribución proporcional de los grupos por XSE, lo que sugiere una exposición similar al agente cn diferentes grupos etarios, sin que se observe un efecto acumulativo. Este último fenómeno podría deberse a que la respuesta inmune humoral contra $L$. pneumophila sea transitoria, disminuyendo progresivamente después de la exposiciốn al agente, característica que sería compartida con otros patógenos bacterianos que no inducen una respuesta inmune humoral permanente como Salmonella typhi, Vibrio cholera y Mycoplasma pneumoniae. Los pocos casos descritos de neumonia por L. pneumophila en Chile ${ }^{11-14}$ han afectado a adultos y no se han informado casos en niños. Este hecho podría ser explicado tanto por la naturaleza esporádica (no epidémica) de la infección en nuestro medio, como también por la existencia de formas clínicas atípicas o bien asintomáticas, que podrian ser más f́recuentes en niños. Mycoplasma pneumoniae, otro agente causal de neumonia atípica, ha mostrado un comportamiento biológico diferente scgún la edad del individuo afectado, teniendo una expresión clínica más severa en el adulto ${ }^{15}$.

Al analizar la seroprevalencia por grupos socioeconómicos destaca la alta exposición a $L$. preumophila en NSE alto, significativamente más alta que en viros NSE. Por cuanto Legionella pneumophila se transmite principalmente por

\section{Tabla 1}

Seroprevalencia de anticuerpos anti-Legionella pneumophila según nivel socioeconómico en población menor de 20 ajios, Santiago, Chile

\begin{tabular}{lcccc} 
& \multicolumn{3}{c}{ Nivel socioecunomico } \\
& Bajo & Medio & Alto & Global \\
\hline $\mathrm{N}(+) /$ Total & $0 / 40$ & $\mathbf{2 / 4 0}$ & $10 / 40$ & $12 / 120$ \\
Prevalencia & 0 & $\mathbf{5 \%}$ & $25 \%$ & $10 \%$ \\
\hline
\end{tabular}

Tabla 2

Seroprevalencia de anticuerpos anti-Legionella pneumophila según grupo etario y nivel socioeconómico

\begin{tabular}{rcccc}
\hline Edad & Bajo & $\begin{array}{c}\text { Nivel socioeconómlco } \\
\text { Medio }\end{array}$ & Alto & $\begin{array}{c}\text { Prevalencla } \\
\text { Global }\end{array}$ \\
\hline $0.5 \mathrm{a}$ & $0 / 10$ & $0 / 10$ & $0 / 10$ & $0 \%$ \\
$6.10 \mathrm{a}$ & $0 / 10$ & $0 / 10$ & $3 / 10$ & $10 \%$ \\
$11-15 \mathrm{a}$ & $0 / 10$ & $1 / 10$ & $3 / 10$ & $13 \%$ \\
$16-20 \mathrm{~s}$ & $0 / 10$ & $1 / 10$ & $4 / 10$ & $17 \%$
\end{tabular}


aerosoles y, por otra parte, la exposición a sistemas de aire acondicionado ${ }^{16-18}$, como $\mathrm{cl}$ uso de calefactores eléctricos (en oposición a los de gas) para calentar el agua empleada en el aseo personal ${ }^{17,20}$, han sido fuentes de numerosos brotes de la infección, es muy posible que la mayor prevalencia en NSE alto refleje mayor exposición a dichos factores de riesgo. De hecho, los sistemas de aire acondicionado y los calefactores eléctricos sólo han sido intraducidos recientemente en nuestro país, y por su alto costo, incorporados prefercntemente en inmuebles a los que sólo tiene acceso la población de más capacidad económica.

La literatura actual no cuestiona el uso de determinación de anticuerpos anti-L. pneumophila como medio para estudiar la exposición previa a este agente, cuya especificidad es 99\% (utilizando un valor de corte de $1: 64$ ), con sensibilidad cercana a $80 \%{ }^{21,22}$. Sin embargo, si bien el método es útil para estudios epidemiológicos, su interpretación como herramienta para el diagnóstico de infección aguda es más compleja. De acucrdo a los resultados que se comentan y otros previos $^{11}$, en Chile no se observan títulos mayores de 1:128 en poblaciones sanas, sin antecedentes de afecciones respiratorias en los últimos ures meses. De este modo parece válido adoptar como criterio serológico de infección reciente un título igual o mayor a $1: 256$, criterio concordante con el del Centro para Control de las Enfermedades, de Allanta, E.U.A. Esta prueba tiene las ventajas de ser fácil, de rápida ejecución y. además, haber sido validada en nuestra población. si bien debe tencrse presente que ella está muy lejos de ser el ideal, tanto por limitaciones biológicas como metodológicas. Desde el punto de vista biológico, conviene recordar que hasta $25 \%$ de los pacientes infectados pueden no desarrollar respuesta humoral inmune y, cn aquellos en que ésta ocurrc, puede tardar hasta tres meses (promedio dos semanas) en hacerse delcctable ${ }^{3}$; desde el punto de vista metodológico, como en la mayoría de las pruebas de inmunofluorescencia, la sensibilidad y la especificidad de ésta también dependen en gran parte del entrenamiento de quien realiza la lectura; por otro lado los antígenos de supcrficie de L. pneumophila, utilizados para efectuarla, comparten deterninantes antigénicos con los lipopolisacáridos de bacierias del género Pseudomonas y Bacteroides ${ }^{23,24}$. Los resultados expuestos sugicren la conveniencia de em. plear pruebas complementarias para el diagnósíco de infección aguda, tales como antigeno en orina, inmunofluorescencia directa o cultivo de muestras de secreciones respiratorias ${ }^{21}, 22$; la detección direcla de Legionella pneumophila mediante inmunofluorescencia, cn muestras obtenidas por lavado brocoalveolar, ha demostrado ser una técnica con alta sensibilidad y especificidad (T. Lobos, R. Moreno, datos no publicados).

\section{Resumen}

Se evaluó la exposición previa a Legionella pneumophila de un grupo de 120 sujetos chilenos, sanos, menores de 20 años, estratificados según nivel socioeconómico. Las muestras de sucro fucron analizadas, para la detección de anticuerpos de Legionella pnetumophila serogrupos 1 a 6 , por técnica de inmunofluorescencia indirecta considerando positivos títulos iguales o mayores a 1:64. Doce de los 120 sujetos fueron positivos (10\%); se observaron diferentes grados de seroprevalencia según niveles sociocconómicos: 0/40 para el más bajo; $2 / 40(5 \%)$ en el nivel medio y $10 / 40(25 \%)$ alto. El mayor lítulo registrado fue 1:128. El nivcl sociocconómico alto está asociado con la prevalencia más alta de anticuerpos anti-Legionella pneumophila en población chilena.

(Palabras clave: Legionella pneumophila, fluorcscencia, inmunocnsayo, anticuerpos, prevalencia.)

\section{Referencias}

1. Fraser DW, Tsai TR, Orenstein W y col.: Legionaires disease: description of an epidemic of pneumonia. X Engl J Med 1977; 297:1189-1197.

2. MfDade JE. Shepard CC, Fraser DW y col. Legionaires disease: isolation of a bacterium and demonstration of its role in other respiratory diseases. N Lingl I Med 1977; 297: 1197-1203.

3. Thornsberry C, Baiows A, Feetey JC y cal (eds.): Le. gionella. Proceedings of the 2nd International Sym. posium. American Society for Microbiology. Washington, D.C. 1984 .

4. Resner $E D$, Helms CM, hierholeer WJ y col.: Legionarics disease in pneumonia patjents in Iowa. A re. Irospective seroepidemiologic study, 1972-1977. Ant Intern Med 1979; 90: 603-606.

5. Foy HM, Broome CV, Hayes PS, y col:: Legionaires disease in a prepaid medical-care group in Seatile 1965. 75. Lancet 1979; i: 767.770. 
6. Andersen $R D$, Lauer $B A$, fraser $D W$ y col.: Infections with Legionella pneumophila in children. J Infect Dis 1981; 143:386-389.

7. Muldoon $R L$, Jaecker $D L, K$ iefer $H K$ : Legionaires discase in children. Pediatrics 1981; 67: 329-332.

8. Mundel $G$, Goldberg A. Boldur / y col:- Legionaires disease in Israel: serological evidence of childhood infection. Isr J Med Sci 1983; 19:380-382.

9. Orenstein WA, Overturf GD, Leedom $J M$ y col: : The frecuency of Legionella infection prospectively detcr. mined in children hospitalized with pneumonia. J Pediatr 198 1: 99: 403-406.

10. Storch $G$, Hayes $P S$, Hill $D L$ y col.: Prevalence of an. ribodies to Legionella preumophila in middle-aged and elderly Americans. J Infect Dis 1979; 140:784-788.

11. Lobos T, Moreno B, Carstens $M$ y col.: Seroprevalencia de Legionella preumophila en Santiago de Chile. Rev Med Chile; 1993; 121:1123-1127.

12. Soto PF. Ncumonitis por Legionella: comunicación de dos casos. Entermedacs Respiratorias y Cirugía Turácica (Santiago, Chile) 1988; 4: 51.

13. Gonzalez $P$, Vicencio $M$, Maldonado $A$. Neumonia por Legionella: primera comunicación de un caso diagnostjeado en Chile. Enfermedades Respiratorias y Cirugia Torácica (Santiago, Chile) 1987: 3: 366-371.

14. Carstens $M$, Muñoz V, Labarca $J$ y col.: Legionelosis aguda, caso clínico. Libro de resúmenes del XXV Congreso Chilcno de Enfermedades Respiratorias, Santiago, Ocrubre $1992 ; \mathrm{p} 76$.

15. Cherry J: Mycoplasma and Ureaplasma Infections. En: Pediatrics Infectious Diseases, Fiegin $y$ Cherry cds. $2^{\text {od }}$ edition, WB Saunders Co, Philadelphia, 1987; pp 1896 . 1924.
16. Dondere $T J$, Rendtorff $R C$, Mallison $G F$ y col: An oubreak of legionaires disease associaled with a contaminated air-conditioning cooling tower. N Engl I Med 1980; 302:365-370.

17. Cordes $L G$, froser $D W$, Skaliy $P$ y col: Legionaires disease outbreak al an Atlanta, Georgia, country club; evidence for spread from an evaporative condenscr. Am J Epidemiol 1980; 111: 425-431.

18. Johnson JT, $Y_{t} V$, Best $M G$ y col.: Nosocomial legionellosis in surgical patients with head and neck cancer: implications for epidemiological reservoir and mode of transmission. Lancet 1985; ii: 298 300 .

19. Alary $M$, Joly $J R$ : Risck factors for contaminalion of domestic hot water systems by Legionella. Appl Environ Microbiol 1991; 57:2360-2367.

20. Lee TC, Stout JE, $Y_{u} V L$ : Factors predisposing to $L e$ gionella pneumophila colonization in residential water systems. Arch Environ Health 1988: 43:59-62.

21. Edetstein PI, Meyer KD: legionaires discase: a teview. Chest 1984; 85: 114-120.

22. Zuravleff JJ, Yu VI, Shonard JW y col: Diactnusis of legionaites diseasc. An update of laboratory methods with emphasis on isolation by culture. JAM $\Lambda$ 1983; $250 \div$ 1981-1985.

23. Collins $M T$, MCDonald J, Hoiby $N$ y col: Agglutinating antibody titers to members of the family Legionelaceae in cystic fibrosis patients as a result of cross-reacting antibodies 10 Pseudomona aeruginosa. J Clin Microbiol $1984 ; 19: 757-62$

24. Katz SM, Holsciaw DS: Scrum antibodics to legionella pnewnophilo in patients with cystic fibrosis. JAMA 1982; 248: 2284-2288.

\section{AVISO A LOS AUTORES}

Con el objeto de dar prioridad a los trabajos de investigación, en vista de las limitaciones de espacio de la Revista Chilena de Pediatría, el Comité Editorial ha acordado restringir la impresión de casos clínicos a un máximo de dos por cada número. 